\title{
Second Order Semi-Discrete Scheme for the Solution of Flow in a Venturi Channel
}

\author{
Susantha Dissanayake ${ }^{1}$ Roshan Sharma ${ }^{1}$ Bernt Lie $^{1}$ \\ ${ }^{1}$ Department of Electrical Engineering, IT and Cybernetics, University of South-Eastern Norway, Porsgrunn, Norway, \\ \{roshan. sharma, bernt. lie\} dusn. no
}

\begin{abstract}
In many different applications, a Venturi channel is used as a tool to compute fluid flow rates. The Saint-Venant equation is a hyperbolic type Partial Differential Equation (PDE) which can be used to model fluid flows through a Venturi channel. The suitability of the $2^{\text {nd }}$ order Kurganov-Petrova (KP) scheme to solve the hyperbolic PDE for fluid flow in the Venturi channel is studied. A laboratory Venturi rig established at the University of South-Eastern Norway (USN) is used to measure the Steady State (SS) fluid levels along the channel. In this paper, the simulated results are compared with the experimental data. In addition, the simulation results obtained with the second order scheme for solving the SaintVenant equations are compared with a $1^{\text {st }}$ order numerical scheme. The Froude number for the flow is calculated to check the flow regime changes: from a subcritical flow to a supercritical flow in the Venturi section of the channel. The $2^{\text {nd }}$ order KP scheme is found to be a suitable numerical scheme which can be used to discretize hyperbolic PDEs.
\end{abstract}

Keywords: Semi-discrete KP scheme, Venturi rig, drill mud flow

\section{Introduction}

The Kurganov-Petrova (KP) scheme is a $2^{\text {nd }}$ order numerical scheme, which is developed to discretize Hyperbolic Partial Differential Equations (PDEs) in spatial directions (Kurganov and Petrova, 2007). In the development of the KP scheme, the local speed of discontinuity propagation is taken into account [2]. Suitability of the KP scheme to simulate flows of water in a reach of a river has been tested by the authors of this paper (Sharma, 2015; Vytvytskyi et al., 2015; Dissanayake et al., 2016). Here, we consider the usefulness of the $2^{\text {nd }}$ order KP scheme to solve the Saint-Venant equation for fluid flow through a Venturi channel. The simulated results are compared to the experimental data obtained from a Laboratory Venturi channel established at the University of South-Eastern Norway (USN). Moreover, the simulation results with the $2^{\text {nd }}$ order KP scheme is compared with a $1^{\text {st }}$ order scheme. Based on the case study, the usefulness of the KP scheme to solve hyperbolic PDEs for fluid flow through the venturi channel is assessed.
In the Finite Volume Methods (FVM), the properties of the flux are averaged in each Control Volume (CV) (Versteeg and Malalasekera, 2007). A linear reconstruction based on the flux property average of CVs, causes discontinuity at the cell interfaces giving rise to the Riemann problem (Kurganov and Tadmor, 2000); the discontinuity causes two different property values for a single interface of a CV and the uniqueness of solution is lost. Subsequently, this may lead to oscillation in the final solution. In order to deal with such discontinuity problems, several numerical techniques have been developed. Various Riemann solvers can be used to address the problem but they usually consist of a number of intermediate calculations to compute a single value for the $\mathrm{CV}$ interface (Kurganov and Petrova, 2007). Due to the number of intermediate calculations involved, Riemann solvers are computationally expensive with slow convergence. The $2^{\text {nd }}$ order KP scheme is a Riemann free solver, which is recognized as an appropriate scheme to address such discontinuity problems with reasonable accuracy and fast convergence (Kurganov and Petrova, 2007).

Use of a Venturi channel as a tool to compute flow rates is well known in many industries, e.g., in oil drilling. The downhole pressure of an oil wellbore is considered as a crucial parameter to be controlled in the drilling operations. Abrupt changes of the pressure of an oil-well might cause minor to major fractures in the wellbore. Such a situation might result in an uncontrolled reduction of oilwell pressure. Subsequently, if the oil well pressure decreases below the formation pore pressure, an unwanted flow of formation fluid into the wellbore occurs. Such a phenomenon is referred to as a kick (Berg et al., 2015). A critical escalation of a kick condition may lead to blow-out from oil wells (Hauge and Øien, 2012). Hence, the kick is a key parameter to observe when drilling is in progress.

Kick is directly related to the drilling fluid circulation. Thus, kick can be identified by applying mass balance to the drilling fluid circulation (Berg et al., 2015). This balance of drilling fluid inflow and outflow of a wellbore can be expressed as follows,

$$
\frac{d m}{d t}=\dot{m}_{\mathrm{i}}-\dot{m}_{\mathrm{e}}
$$

Here, $\dot{m}_{\mathrm{i}}$ is the mass flow rate of drilling fluid injected into the well (influent), $\dot{m}_{\mathrm{e}}$ is the mass flow rate of the fluid 
flowing out of the well (effluent) and $m$ is the mass of fluid in the well. For the detection of the kick and loss, it is important to accurately measure the flow rate of the fluid flowing out of the well. In oil industries, the use of a measuring paddle in an open channel is a typical/conventional way of measuring the flow rate out of the well. One of the alternatives is to use an open channel system such as the Venturi flume/Venturi channel for return drill fluid flow measurement (Berg et al., 2015).

In this paper, the $2^{\text {nd }}$ order KP scheme is used to discretize the Saint-Venant equation spatially. The objective is to check the suitability of the $2^{\text {nd }}$ order KP scheme to solve hyperbolic type PDEs based on the case study of the solution of the flow in the Venturi channel. The considered laboratory Venturi channel has level sensors in fixed positions which provides the SS fluid level for a known volumetric flow rate. The set of experimental data is compared to the simulation results with the $2^{\text {nd }}$ order KP scheme. In the latter part of the study, both experimental data and the $2^{\text {nd }}$ order simulation results are compared to a set of $1^{\text {st }}$ order simulation results which have been published previously (Agu, 2014).

This paper is arranged as follows. The Venturi channel and its use for flow rate estimation is discussed in section 2. A detailed description of the laboratory Venturi rig is given in the same section, with a set of ground floor tanks, upstream pipe, and the Venturi channel itself. A basic introduction to the Saint-Venant equation together with the $2^{\text {nd }}$ order KP scheme is given in section 3 . The model of the laboratory Venturi channel is simulated in section 4. Simulation parameters, variables, and quantities are tabulated in the same section. Section 5 provides a discussion of results: how the simulated results compare with the experimental data. Moreover, simulation results of a $1^{\text {st }}$ order numerical scheme (Agu et al., 2015) are discussed, and the percentage errors of the two different methods are given. Finally, some conclusions are drawn in section 6 .

\section{The Venturi Channel}

The Venturi channel or Venturi flume is an open channel system. The Venturi channel holds a section where the width narrows; the Venturi section. When fluid flows through a Venturi channel, the fluid level is decreased in the Venturi section due to an acceleration of the flow velocity. In the SS fluid flow, a correlation between volumetric flow rate and the fluid height can be derived. Consequently, either volumetric flow rate or fluid height is computed. Typically, the fluid flow rate is calculated based on the level measurements. The Venturi channel has two different names based on applications. The Venturi tube is used for relatively smaller flow (e.g. $\mathrm{mm}^{3} / \mathrm{s}$ ), while the Venturi channel is used for large scale flows with up to millions of cubic meter per hour units. The Venturi tube was developed by G.B. Venturi based on the Bernoulli principle,

$$
\frac{v^{2}}{2}+g h+\frac{p}{\rho}=\text { constant }
$$

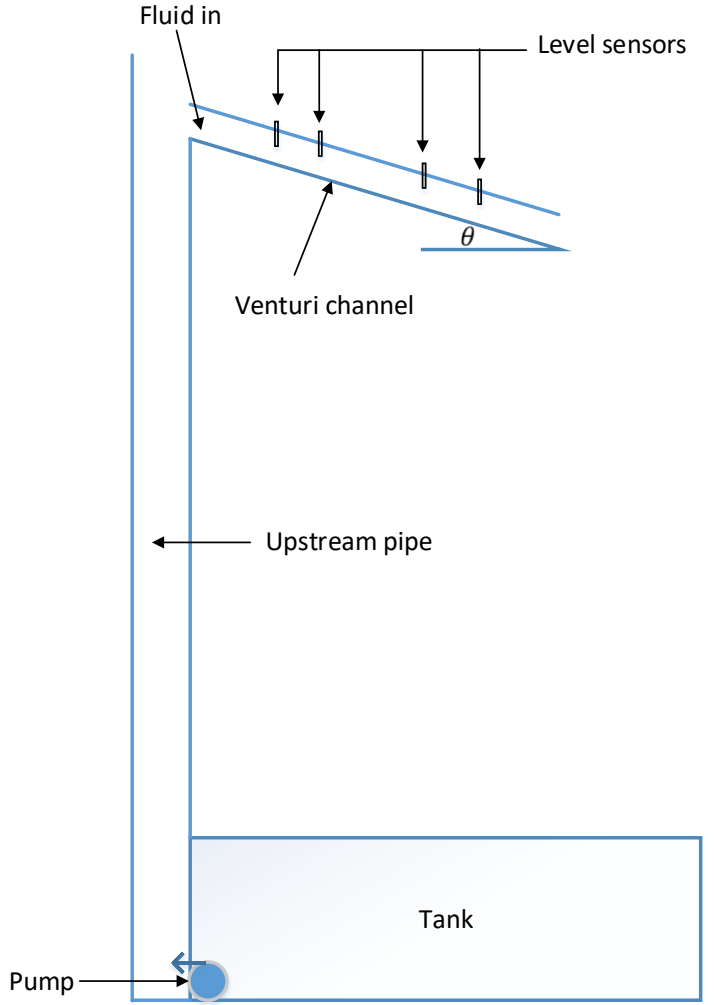

Figure 1. Laboratory Venturi rig.

Here, $v$ denotes the velocity of the fluid, $g$ is the acceleration of gravity, $h$ is the height elevation from the reference plane, $p$ is the pressure of the fluid, and $\rho$ denotes the density of the fluid.

In the laboratory Venturi rig, the volumetric flow rate is an adjustable variable. The SS fluid height is measured by level sensors at fixed positions. The USN Venturi rig is designed for doing experiments on the flow of drill mud: more precisely, experiments on flow of artificial drill mud . An artificial drill mud is a substitute fluid with properties that match with the real drill mud.

The complete set-up has ground tanks for storing artificial drill mud. The system has an upstream pipe which pumps fluid from the tanks and up to some elevation $\left(2^{\text {nd }}\right.$ floor). At the end of the upstream pipe, the Venturi channel is attached. A schematic diagram of the complete setup of the Venturi rig at USN is illustrated in Figure 1.

A detailed sketch of the Venturi channel is given in Figure 2. The dimensions of the laboratory Venturi channel are tabulated in Table 1. The total length of the Venturi channel is $3.7 \mathrm{~m}$. 
Table 1. Dimensions of the laboratory venturi channel

\begin{tabular}{lll}
\hline Symbol & Description & Values \\
\hline$B_{T}$ & Top width of entrance to the converging section & $0.455[\mathrm{~m}]$ \\
$b_{0}$ & Bottom width of entrance to the converging section & $0.2[\mathrm{~m}]$ \\
$b_{* 0}$ & Bottom width of exit from the converging section & $0.1[\mathrm{~m}]$ \\
$H$ & Total height of the channel & $0.35[\mathrm{~m}]$ \\
$\alpha$ & Angle of the inclined sides of the channel & $70^{0}$ \\
$L_{a}$ & Length of the upstream section & $2.95[\mathrm{~m}]$ \\
$L_{b}$ & Length of the converging section & $0.15[\mathrm{~m}]$ \\
$L_{c}$ & Length of the throat section & $0.2[\mathrm{~m}]$ \\
$L_{d}$ & Length of the diverging section & $0.15[\mathrm{~m}]$ \\
$L_{e}$ & Length of the downstream section & $0.25[\mathrm{~m}]$ \\
$\theta$ & Angle of channel bed & $0.08^{0}$ \\
\hline
\end{tabular}

\section{Governing Equations and the $2^{\text {nd }}$ Order KP Scheme}

\subsection{Governing Equations}

The Saint-Venant equation/Shallow Water Equation is a hyperbolic type PDE, which has versatile use in fluid dynamics: many different fluid dynamic applications such as fluid flows in open channels, water flow in the rivers to estimate wave propogation, compute tsunmai wave, etc. (Sharma, 2015; Vytvytskyi et al., 2015; Dissanayake et al., 2016). The Saint-Venant equation is given in Equation 3.

$$
\frac{\partial U}{\partial t}+\frac{\partial F}{\partial x}=S
$$

Here, $U$ stands for fluid property such as the flow rate and fluid level, $F(U)$ represents physical fluid flux and $S$ is a source term. $x$ and $t$ are spatial and time cordinates respectively. More precisely, the Saint-Venant equation with source term is given as (Sharma, 2015),

$$
\begin{gathered}
\frac{\partial U(x, t)}{\partial t}+\frac{\partial F(x, t, U)}{\partial x}=S(x, t, U), \\
U=(A, Q)^{T} \\
F=\left(Q, \frac{Q^{2}}{A}+g I_{1} \cos (\theta)\right)^{T} \\
S=\left(0,-g I_{2}+g A\left(S_{0}-S_{f}\right)\right) .
\end{gathered}
$$

Here, $A$ is the cross-sectional area, $Q$ is the volumetric flow rate and $g$ is acceleration due to gravity. The $S$ term reflects the source terms such as the expressions of friction terms which give resistance against the fluid flow. $I_{1}$ and $I_{2}$ in Equations 6 and 7 are functions of the geometry of the channel. The term $g I_{1}$ gives the hydrostatic force in the cross-sectional area of the flow and $I_{1}$ is expressed as,

$$
I_{1}=h^{2}\left(\frac{W}{2}+h \frac{S_{L}}{3}\right) \text {. }
$$

Here $W$ is the width of the base of the channel and $S_{L}$ is the slope of the side wall (the laboratory Venturi rig is trapezoidal). If the channel is rectangular, then $S_{L}$ become zero. $h$ is fluid level. $I_{2}$ in Equation 7 represents the pressure force in the fluid volume, which occurs due to channel width and slope variations along the axial direction.

$$
I_{2}=h^{2}\left(\frac{1}{2} \frac{d W}{d x}+h \frac{h}{3} \frac{d S_{L}}{d x}\right) .
$$

$S_{f}$ in Equation 7 is friction term and $S_{0}$ stands for bed slope which is expressed as,

$$
S_{0}=-\frac{\partial B}{\partial x}
$$

Here $B$ stands for the bottom elevation. For Newtonian fluids, the friction term $S_{f}$ in Equation 7 is calculated as (Agu et al., 2015),

$$
S_{f}=v|v| n_{\mathrm{M}}^{2} R_{\mathrm{h}}^{-4 / 3} .
$$

Here $n_{\mathrm{M}}$ is Manning's roughness coefficient. $v$ is the velocity of the fluid and is calculated as,

$$
v=\frac{Q}{A}
$$

$R_{\mathrm{h}}$ in Equation 11 refers to hydraulic radius and is expressed as in Equation 13.

$$
R_{\mathrm{h}}=\frac{A}{P_{\text {wet }}} .
$$

$P_{\text {wet }}$ is the wetted perimeter which is illustrated in Figure 3.

$P_{\text {wet }}$ in Equation 13 is the sum of all lengths of wetted surface by the fluid, which is expressed as,

$$
P_{\mathrm{wet}}=\sum_{i=0}^{\infty} l_{i} .
$$

Here $l_{i}$ is the length of each surface which has contact with the fluid flow in the channel. 


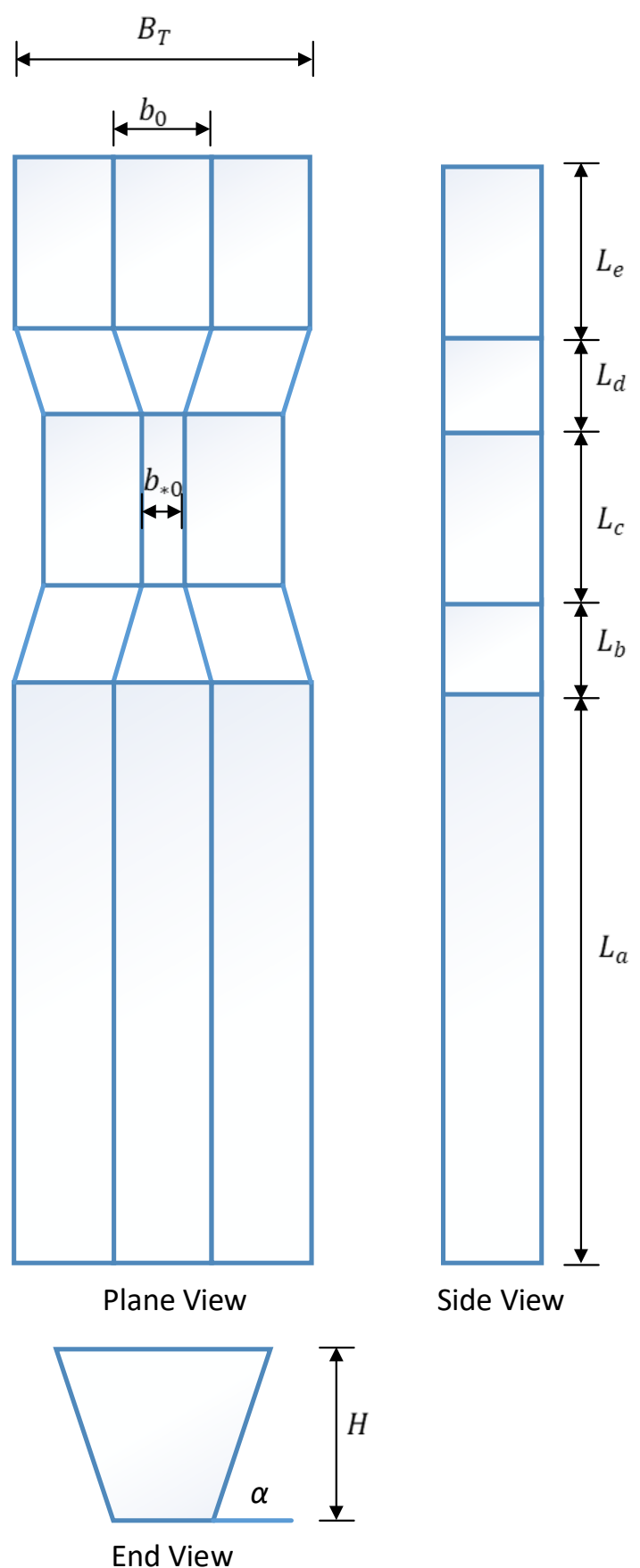

Figure 2. Illustration of the Laboratory Venturi channel.

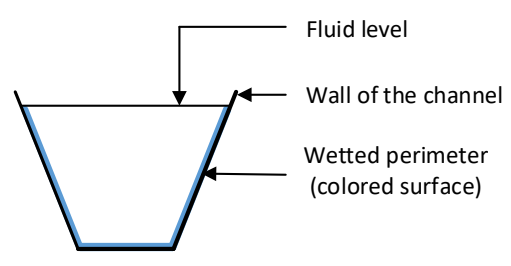

Figure 3. The wetted perimeter of a trapezoidal channel.
Many common fluids are assumed as Newtonian, e.g., water. A Newtonian fluid is a fluid for which the viscous stresses developed due to the flow of fluid are linearly proportional to local strain rates or rate of change of deformation (Versteeg and Malalasekera, 2007). The drilling fluid however is a non-Newtonian fluid.

\subsection{Non-Newtonian Fluid}

Non-Newtonian fluids are fluids for which the viscous stresses developed due to the flow of fluid are not linearly proportional to the rate of deformation. Polymers, paints, and Oobleck ${ }^{1}$ are common examples of non-Newtonian fluids. Drill mud consist of fines of drill cuttings in a mixture of liquid, and is exhibits a non-Newtonian fluid behvaiour.

Therefore, fluids used in our simulations are considered as non-Newtonian fluid. The friction term in the Saint Venant equation is modified to account for more types of fluids. Subsequently, the Venturi rig simulation is extended to the non-Newtonian fluid.

Shear stress, $\tau$, is a component of stress applied on the material cross-sectional area. Shear stress is written as,

$$
\tau=\frac{F}{A}
$$

Here, $F$ refers to forces applied on the cross sectional area $A$. For a flowing fluid, the shear stress becomes,

$$
\tau=\mu\left(\frac{\partial v}{\partial x}\right)
$$

Here $\mu$ is the dynamic viscosity of the fluid. The term $\frac{\partial v}{\partial x}$ is the velocity gradient. $x$ signifies the spatial coordinate.

For non-Newtonian fluid, shear stress is derived by applying the momentum balance. Then $\tau$ is written as in Equation 17, the well-known Herschel-Buckley model.

$$
\tau=\rho g S_{f}\left(R_{\mathrm{h}}-h\right)=\tau_{h}+K\left(\frac{\partial u}{\partial x}\right)^{n}
$$

In Equation 17, $\rho$ signifies the density of the fluid and $g$ denotes the acceleration due to gravity. $h$ is height above the reference plane. $K$ is the consistency index, $u$ in the equation stands for the velocity component in the positive $x$ direction, $\tau_{h}$ is yield stress and $n$ is the flow index. For $n<1$, the fluid is shear thinning. If $n>1$, the fluid is shear thickening. If $n=1$ and $\tau_{h}=0$, Equation 17 reduces to Newtonian model (Agu, 2014).

For non-Newtonian fluids, internal frictional shearing stress has a major impact. Then from Equation $17, S_{f}$ is derived as (Agu et al., 2015),

$$
S_{f}=\frac{K}{4 \rho g R_{\mathrm{h}}}\left(\frac{|v|}{h} \frac{1+2 n}{n}\right)^{n} .
$$

${ }^{1}$ Oobleck is corn flour in water. The name originates from a storybook: Bartholomew and the Oobleck by Dr. Seuss (Theodor Geisel). 


\subsection{The $2^{\text {nd }}$ Order KP Scheme}

The $2^{\text {nd }}$ order KP scheme is used to discretize the SaintVenant equation. The $2^{\text {nd }}$ order KP scheme is semidiscrete in nature, and is used in the spatial discretization of the model equations (Kurganov and Petrova, 2007). Spatial discretization of the Saint-Venant equation results in a set of Ordinary Differential Equations (ODEs). Different time integrators can be used to solve such ODEs. However, the chosen time integrator should essentially be of order equal to or lower than the order of the spatial discretization to attain fast convergence. Also, (Dissanayake et al., 2016) found that fixed step length solvers are more suitable for solving such a set of ODEs. The ODEs resulting from the spatial discretization are of $2^{\text {nd }}$ order. Therefore, a $2^{\text {nd }}$ order Runge-Kutta (RK2) method is used as a time integrator.

The set of ODEs generated by the spatial discretization of the Saint-Venant equation using the KP scheme is written as (Sharma, 2015),

$$
\begin{gathered}
\frac{d}{d t} \bar{U}_{j}=-\frac{H_{j+\frac{1}{2}}(t)-H_{j-\frac{1}{2}}(t)}{\triangle x} \\
H_{j+\frac{1}{2}}(t)=\frac{a_{j+\frac{1}{2}}^{+} F\left(U_{j+\frac{1}{2}}^{-}, B_{j+\frac{1}{2}}\right)-a_{j+\frac{1}{2}}^{-} F\left(U_{j+\frac{1}{2}}^{+}, B_{j+\frac{1}{2}}\right)}{a_{j+\frac{1}{2}}^{+}-a_{j+\frac{1}{2}}^{-}} \\
+\frac{a_{j+\frac{1}{2}}^{+} a_{j+\frac{1}{2}}^{-}}{a_{j+\frac{1}{2}}^{+}-a_{j+\frac{1}{2}}^{-}}\left(U_{j+\frac{1}{2}}^{+}-U_{j+\frac{1}{2}}^{-}\right)
\end{gathered}
$$

$$
\begin{aligned}
H_{j-\frac{1}{2}}(t) & =\frac{a_{j-\frac{1}{2}}^{+} F\left(U_{j-\frac{1}{2}}^{-}, B_{j-\frac{1}{2}}\right)-a_{j-\frac{1}{2}}^{-} F\left(U_{j-\frac{1}{2}}^{+}, B_{j-\frac{1}{2}}\right)}{a_{j-\frac{1}{2}}^{+}-a_{j-\frac{1}{2}}^{-}} \\
& +\frac{a_{j-\frac{1}{2}}^{+} a_{j-\frac{1}{2}}^{-}}{a_{j-\frac{1}{2}}^{+}-a_{j-\frac{1}{2}}^{-}}\left(U_{j-\frac{1}{2}}^{+}-U_{j-\frac{1}{2}}^{-}\right)
\end{aligned}
$$

where $a_{j \pm \frac{1}{2}}^{ \pm}$are the one-sided local speeds of wave propagation and $U_{j \pm \frac{1}{2}}^{ \pm}$are property fluxes at indexed positions.

\section{Simulation Setup}

The model of the laboratory Venturi channel is simulated for the flow of artificial drill mud. Important simulation parameters are summarized in Table 2. MATLAB ${ }^{2}$ is used as a simulation software.

\section{Results and Discussion}

Width variation along the length of the Venturi channel is plotted in Figure 4. According to the dimensions of the laboratory Venturi channel (Table 1), the width of the

${ }^{2}$ MATLAB R2014a
Table 2. Simulation Parameters.

\begin{tabular}{ll}
\hline Length of the Venturi channel & $3.7[\mathrm{~m}]$ \\
Density of the fluid & $1109\left[\mathrm{kgm}^{-3}\right]$ \\
Acceleration due to gravity & $9.81\left[\mathrm{~ms}^{-2}\right]$ \\
Channel side angle & $70^{0}$ \\
Channel bed angle & $0.08^{0}$ \\
Volumetric flow in & $0.00451\left[\mathrm{~m}^{3} \mathrm{~s}^{-1}\right]$ \\
Volumetric flow out & $0.00451\left[\mathrm{~m}^{3} \mathrm{~s}^{-1}\right]$ \\
Number of CVs & 50 \\
Time step $(\triangle t)$ & $0.02[\mathrm{~s}]$ \\
\hline
\end{tabular}

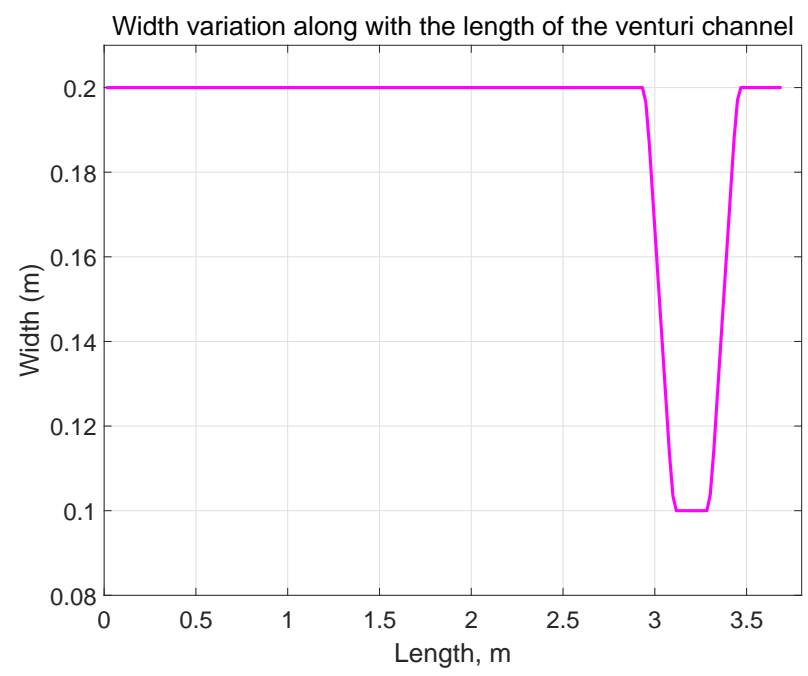

Figure 4. Width variation in the Venturi rig as a function of the length of the channel.

channel varies gradually between $0.2 \mathrm{~m}$ and $0.1 \mathrm{~m}$ at the throat section of the channel. The SS fluid level along the whole length of the Venturi channel obtained using the KP scheme is shown in Figure 5.

According to Figure 5, the initial fluid level is about $0.076 \mathrm{~m}$ from the reference plane (which is the bottom of plane of the channel). The SS fluid level decreases in the Venturi section: after $3 \mathrm{~m}$ along the channel, the fluid level starts to decrease and continues to decrease until position ca. $3.4 \mathrm{~m}$ along the Venturi channel. According to the dimensions of the Venturi channel (Table I), the Venturi section starts at position $2.95 \mathrm{~m}$. In other words, the width of the channel is gradually reducing from $0.2 \mathrm{~m}$ to $0.1 \mathrm{~m}$ after $2.95 \mathrm{~m}$ from the starting point of the Venturi channel. Downstream from the Venturi section: after 3.45 $\mathrm{m}$, the channel width is back again to $0.2 \mathrm{~m}$. Downstream from the Venturi section, the level of the fluid remains constant: more or less steady level throughout the rest of the length: $3.45 \mathrm{~m} \leq$ Length $\leq 3.6 \mathrm{~m}$. Visual observation and level sensor readings support these changes in flow behavior. Experimental data from the Venturi rig is plotted with the simulated SS fluid level, in order to check the accuracy of the simulation. 


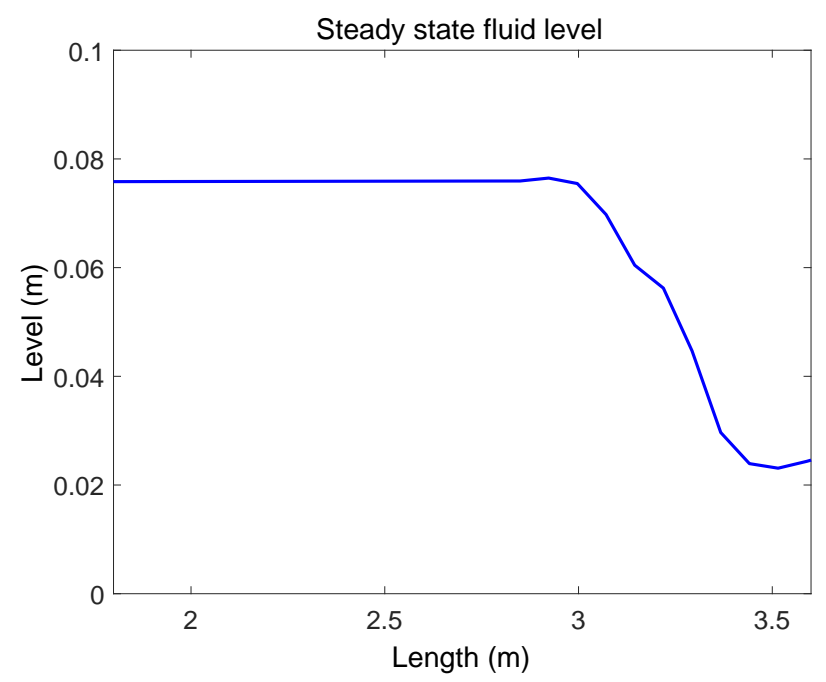

Figure 5. Steady State fluid level in the Venturi channel using KP scheme.

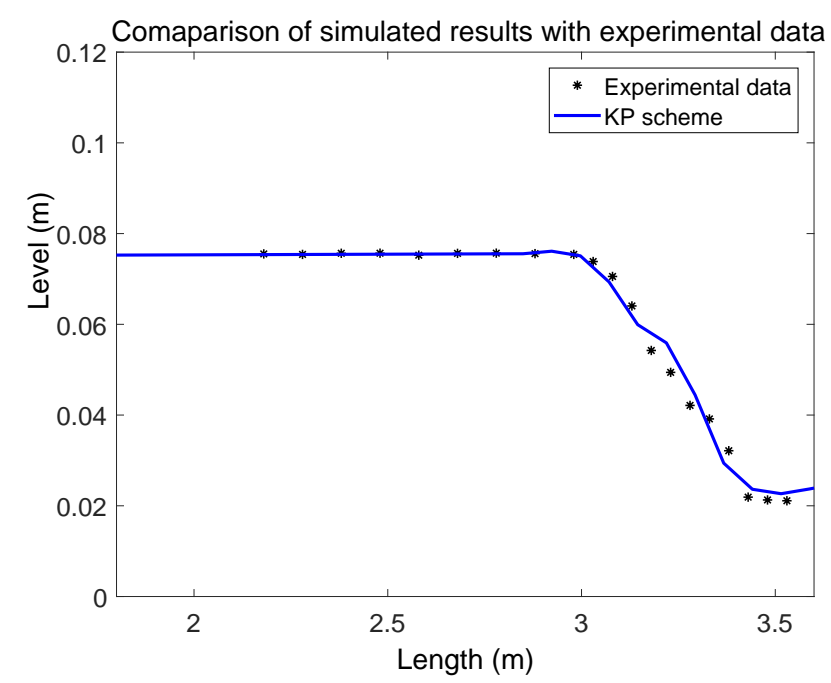

Figure 6. Comparison of simulation results with KP scheme with experimental data.

\subsection{Comparison of Simulated Results With Experimental Data}

The laboratory Venturi rig has a few level sensors which can measure the fluid level at several positions in the Venturi channel. Simulated data and experimental data have been plotted and compared in this section. Figure 6, shows the simulation results together with the experimental data.

In a previous work by (Agu et al., 2015), a similar study of the exact same Venturi channel was carried out. In their study, the authors derived the ODEs by the spatial discretization of the Saint-Venant equation with a $1^{\text {st }}$ order scheme. Both the $1^{\text {st }}$ order simulation results [11], and the $2^{\text {nd }}$ order simulation results are plotted together with the experimental data in Figure 7. (Agu et al., 2015) used MATLAB ode solver ode15s in their simulations.

From Figure 7 , both the $1^{\text {st }}$ order and the $2^{\text {nd }}$ order

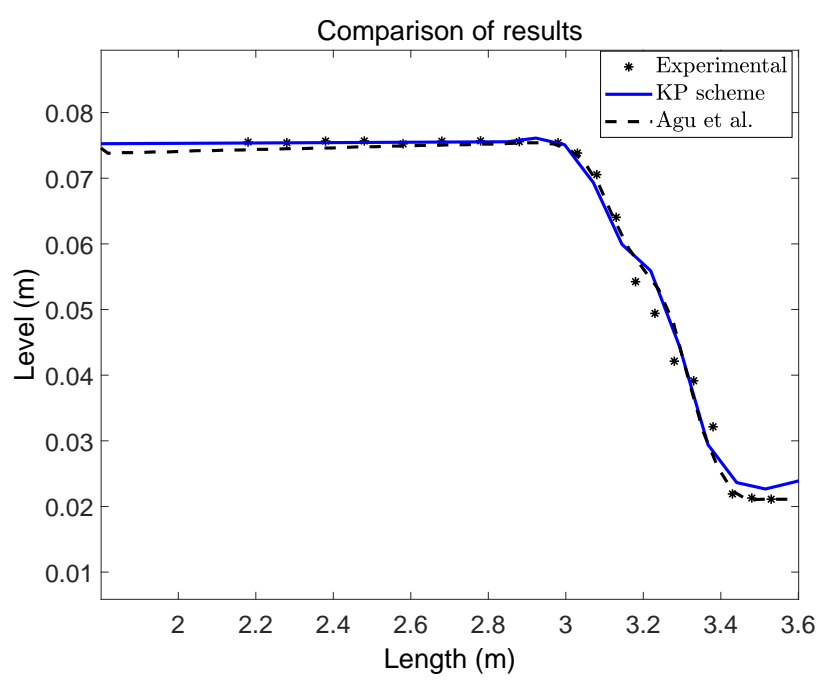

Figure 7. Comparison of the simulation results from $2^{\text {nd }}$ order KP scheme and $1^{\text {st }}$ order scheme with experimental data.

scheme produce similar results at the steady state. In the Venturi section, both schemes follow the similar pattern. However, both schemes show a small deviation from the experimental data. After the Venturi section, the 1st order model approximation is closer to the experimental data; the $2^{\text {nd }}$ order KP scheme shows a small deviation.

\subsection{Error Computation}

Simulated results have only a minor deviation from the experimental data. Fluid level readings have been taken at fixed positions along the Venturi channel. These fixed positions do not necessarily fall at the centers of the grid cells of the FVM. The simulated results have thus been interpolated to compute the values of the fluid level such that they correspond to these fixed positions (where level sensors are placed) and then only compared with the experimental data. These interpolated values and the experimental data are plotted together with the simulated results in Figure 8.

In a similar way, interpolated values and the experimental data are plotted for the study of (Agu et al., 2015) where a first order scheme for spatial discretization is used. Figure 9 shows the interpolated points, experimental data and the simulation of (Agu et al., 2015).

The difference between the interpolated values and the experimental data are the error of the simulated results. Percentage error of the $2^{\text {nd }}$ order KP scheme and the $1^{\text {st }}$ order scheme of (Agu et al., 2015) are plotted in Figure 10.

According to Figure10, the $2^{\text {nd }}$ order KP scheme has negligible percentaged error upstream from the Venturi section: $2.2 \mathrm{~m} \leq$ Level $\leq 3 \mathrm{~m}$. The simulation results of (Agu et al., 2015) exhibit a small deviation for this section of the channel. In the Venturi section both simulated results show more or less similar behavior. However, the simulation of (Agu et al., 2015) has a slightly larger overshoot and undershoot in the Venturi section. The simula- 


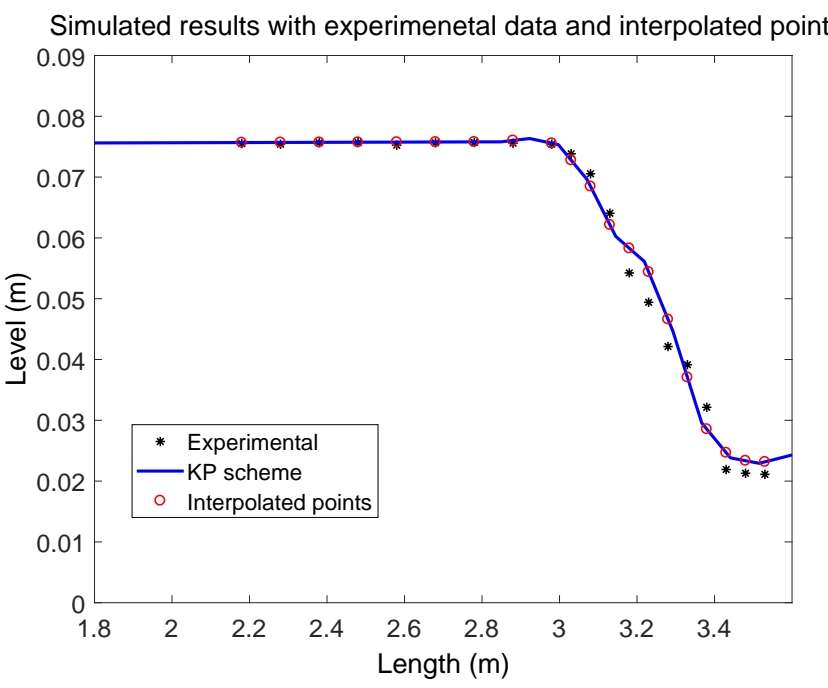

Figure 8. Simulation results with the $2^{\text {nd }}$ order KP scheme, interpolated points, and experimental data.

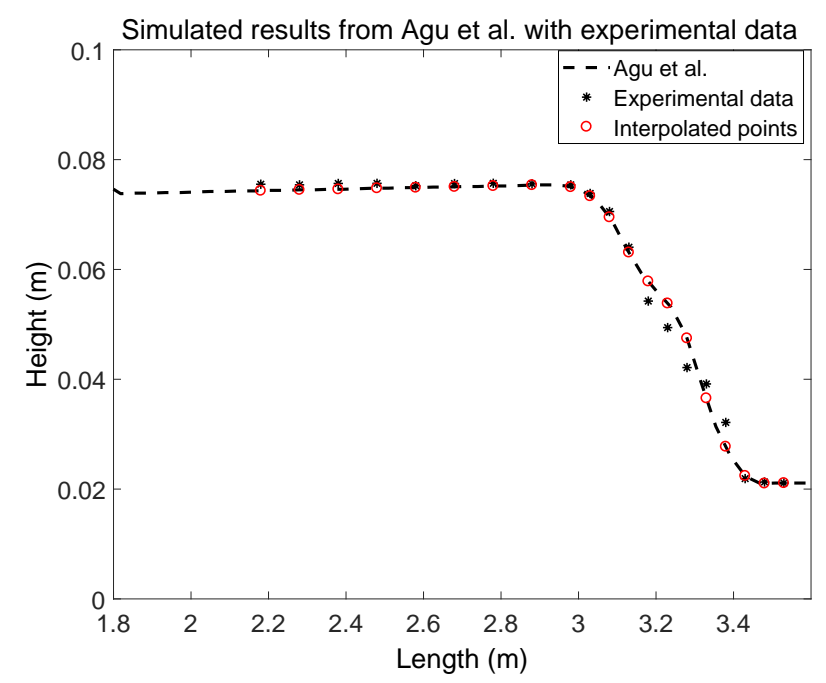

Figure 9. Simulated results of (Agu et al., 2015), experimental data and interpolated points.

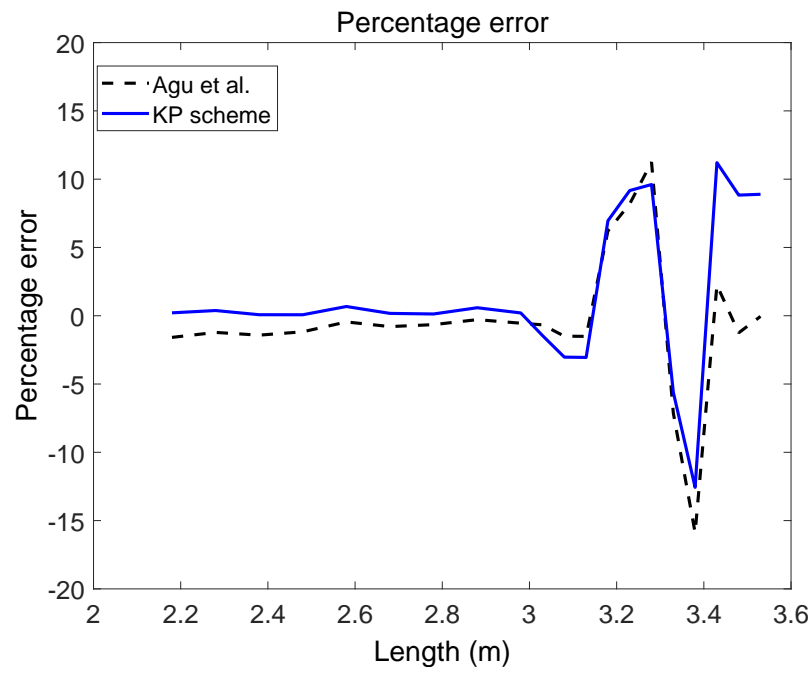

Figure 10. Percentage error for $2^{\text {nd }}$ order KP scheme and the 1 st order scheme of (Agu et al., 2015).

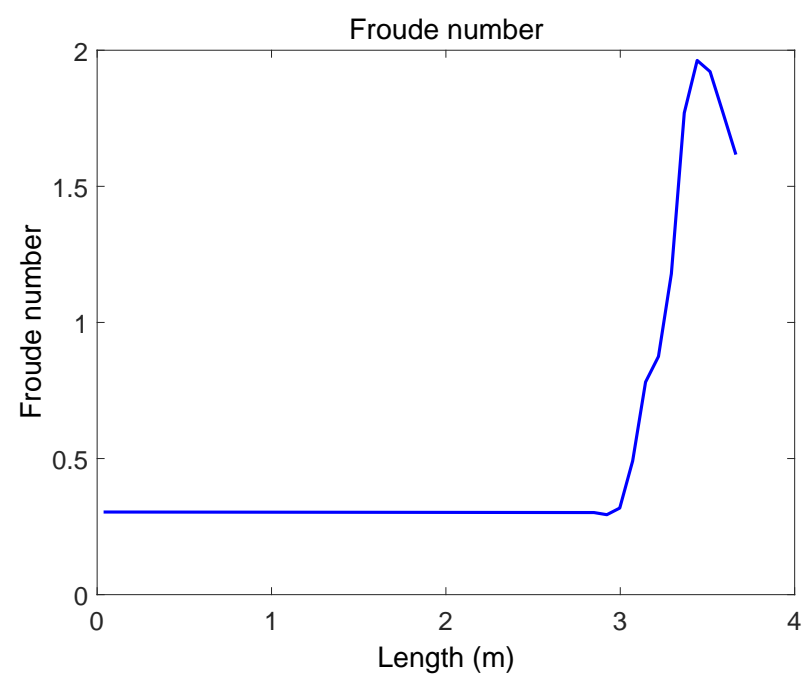

Figure 11. Froude number along the length of the venturi channel.

tion of (Agu et al., 2015) produces a slightly less percentage error after the Venturi section.

As a whole, the KP scheme produces acceptable results for the whole length of the Venturi channel apart from the length: $3.4 \mathrm{~m} \leq$ Length (the part which is not useful and thus not used for the estimation of fluid flow rate).

\subsection{Flow Regimes}

Flow velocity changes along the length of the Venturi channel can be found by computing the Froude number (Fr) which is a dimensionless number that explains the speed-length ratio, and is written as,

$$
\operatorname{Fr}=\frac{u_{0}}{\sqrt{g_{0} l_{0}}} .
$$

Here $u_{0}$ is characteristic flow velocity, $g_{0}$ is characteristic of the external field: more precisely, acceleration of gravity. $l_{0}$ stands for characteristic length. Fr for the current scenario can be written as,

$$
\operatorname{Fr}=\frac{u_{0}}{v} .
$$

Here $v$ is the characteristic fluid propagation velocity and is written as,

$$
v=\sqrt{g h},
$$

where $g$ is gravitational constant and $\mathrm{h}$ is fluid level.The Frode number computed along the Venturi channel is plotted in Figure 11. When Fr increases and becomes greater than 1, this means that the flow changes from subcritical to supercritical. In the Venturi section of the channel, flow becomes supercritical.

\subsection{Flow Rate Calculation}

When the fluid flow is at SS, fluid level is measured. When the flow rate increases, the level increases. Such increment of the height of the flowing fluid increases the wetted 
perimeter. The wetted perimeter has a correlation with the hydraulic radius (Equation 13). According to Equation 2 , total energy in the system is preserved. Based on the known parameters, back calculation can be used to compute volumetric flow rate of the fluid.

\section{Conclusions}

Based on this study, the KP scheme is recognized as a suitable numerical scheme to discretize the Saint-Venant equations, which is a hyperbolic PDE. ODEs resulting from the spatial discretization has a $2^{\text {nd }}$ order of accuracy. Hence when the KP scheme is compare with the $1^{\text {st }}$ order scheme, the KP scheme shows increment in the accuracy. According to the percentage error comparison, it is concluded that increment of the order of the spatial discretization improve the accuracy. Flow regime changes along the Venturi channel is observed with the Frode number. The KP scheme successfully recognized the flow regime change from subcritical to supercritical. For the Venturi section both schemes show deviation from the experimental results. However, the $1^{\text {st }}$ order scheme shows slightly larger overshoot and undershoot. Hence the KP scheme can be used to solve the Saint-Venant equations for the flow through a venturi channel.

\section{Acknowledgements}

The economic support from The Research Council of Norway and Statoil ASA through project no. 255348/E30 "Sensors and models for improved kick/loss detection in drilling (Semikidd)" is gratefully acknowledged. Mr. Cornelius Emeka Agu supplied materials, simulation results, experimental data, etc. This is gratefully acknowledged.

Kindly convey sincere thanks to the other project group members: Janitha Chandimal, Junyang Mao, and Obianuju Ezuka.

\section{References}

C. E. Agu. Model based estimation of drilling mud flow using a venturi channel. Master's thesis, Faculty of Technology, Telemark University College, Porsgrunn, Norway, 2014.

C. E. Agu, B. Lie, and G. Elseth. Simulation of transcritical flow in hydraulic structures. In Proceedings of the 56th Conference on Simulation and Modelling (SIMS 56), number 119, pages 369-375, Linköping University, Sweden, October 7-9 2015. ISBN 978-91-7685-900-1. doi:10.3384/ecp15119369.

C. Berg, A. Malagalage, C.E. Agu, G.-O. Kaasa, and B. Lie. Model-based drilling fluid flow rate estimation using venturi flume. 2nd IFAC Workshop on Automatic Control in Offshore Oil and Gas Production, 48(6):171-176, 2015.

S. Dissanayake, R. Sharma, and B. Lie. Semi discrete scheme for the solution of flow in river tinnelva. In Proceedings of EUROSIM 2016, pages 134-139, Oulu, Finland, September 13-16 2016. IEEE. ISBN ISBN 978-1-5090-4119-0.

S. Hauge and K. Øien. Deep water horizon: Lessons learned for the norwegian petroleum industry with focus on technical aspects. Chemical Engineering Transactions, 26:621-626, 2012. ISSN 1974-9791. doi:10.3303/CET1226104.

A. Kurganov and G. Petrova. A second-order well-balanced positivity preserving central-upwind scheme for the saint-venant system. Communications in Mathematical Science, 5:13360, 2007.

A. Kurganov and E. Tadmor. New high-resolution central schemes for nonlinear conservation laws and convection-diffusion equations. Journal of Computational Physics, 160(1):241 - 282, 2000. ISSN 0021-9991. doi:10.1006/jcph.2000.6459.

R. Sharma. Second order scheme for open channel flow. Technical report, USN Open Archive, University of SouthEastern Norway, 2015. URL http://hdl handle. net $/ 11250 / 2438453$.

H. K. Versteeg and W. Malalasekera. An introduction to computational fluid dynamics. Pearson Education, Upper Saddle River, United States, 2nd edition, 2007. ISBN 9780131274983.

L. Vytvytskyi, R. Sharma, and B. Lie. Model based control for run-of-river system. part 1: Model implementation and tuning. Modeling, Identification and Control, 36(4):237-249, 2015. doi:10.4173/mic.2015.4.4. 